\title{
Comparing the clinical severity of disease caused by enteroviruses and human
} parechoviruses in neonates and infants

Sally Black ${ }^{1}$, Carina Bradley ${ }^{2}$, Florence Y Lai ${ }^{3}$, Savitha Shenoy ${ }^{1}$, Srini Bandi ${ }^{1}$, David J Allen ${ }^{4,5}$, Julian W Tang ${ }^{2,6}$

${ }^{1}$ Leicester Children's Hospital, University Hospitals of Leicester NHS Trust, Leicester, UK ${ }^{2}$ Clinical Microbiology, University Hospitals of Leicester NHS Trust, Leicester, UK

${ }^{3}$ Department of Cardiovascular Science, University of Leicester, Leicester, UK

${ }^{4}$ Virus Reference Department, National Infections Service, Public Health England, London, UK

${ }^{5}$ Pathogen Molecular Biology Department, Faculty of Infectious and Tropical Diseases, London School of Hygiene and Tropical Medicine, UK

${ }^{6}$ Infection, Immunity, Inflammation, University of Leicester, Leicester, UK

\section{Correspondence to: Dr Julian W Tang}

Clinical Microbiology, University Hospitals of Leicester NHS Trust

Level 5 Sandringham Building, Leicester Royal Infirmary

Infirmary Square, Leicester LE1 5WW, UK.

Email: julian.tang@uhl-tr.nhs.uk; jwtang49@hotmail.com

Tel: 0116258 6516. Fax: 01162551949 


\begin{abstract}
Comparison of children hospitalised with enterovirus (EV) or human parechovirus $(\mathrm{HPeV})$ infections of their cerebrospinal fluid (CSF) revealed that $\mathrm{HPeV}$ infections presented with more persistent fever, irritability and feeding problems, more frequent leukopenia and lymphopenia, and higher admission rates to high dependency or intensive care units. As very few $\mathrm{HPeV}$ cases were followed-up, further studies on long-term outcomes are needed.
\end{abstract}

\title{
Word count 60
}




\section{HIGHLIGHTS}

Children infected with $\mathrm{HPeV}$ had more persistent symptoms than those infected with EV

Children infected with HPeV had higher HDU/ICU admissions than those infected with EV

Children infected with $\mathrm{HPeV}$ had more leukopenia than those infected with EV

Children infected with EV had higher CSF white cells than those infected with EV

Further studies are needed to characterise any longer-term EV and HPeV complications 


\section{INTRODUCTION}

Enteroviruses are well-known causes of sepsis in neonates and infants. In recent years, the extent to which parechoviruses may be contributing to neonatal and infant morbidity and mortality has begun to emerge. ${ }^{1-3}$

Enteroviruses (EV) and human parechoviruses $(\mathrm{HPeV})$ are non-enveloped, singlestranded, positive-sense RNA viruses and members of the Picornavirus family. They are common causes of neonatal and infant sepsis, worldwide.

Enteroviruses exist as multiple serotypes, subdivided into various genus, including echoviruses, Coxsackie $\mathrm{A}$ and $\mathrm{B}$ viruses, and the numbered enteroviruses. Human parechoviruses exist in at least 17 genotypes, of which genotypes 1-6 are most commonly found in humans, with genotype 3 being most commonly responsible for sepsis in neonates and infants.

Whilst most episodes of EV and HPeV neonatal and infant sepsis are self-limiting, more severe illness can occur and there are current concerns about longer-term sequelae, particularly in $\mathrm{HPeV}$ infections where there is more significant neurological involvement. Previous studies have found that the clinical presentation of the two viruses are often indistinguishable. ${ }^{1,3}$

Our diagnostic virology laboratory has only relatively recently (since mid-2014) introduced routine testing for parechoviruses as part of our neonatal and infant septic workup. We examined the demographics, laboratory results and clinical notes for paediatric patients admitted with sepsis with laboratory-confirmed human enterovirus (EV) or parechovirus (HPeV) infections of the cerebrospinal fluid (CSF), during Feb 2014 to Aug 2017.

\section{METHODS}


All cases from a 3.5 year period (Feb 2014 to Aug 2017) were selected on the basis of a positive cerebrospinal fluid (CSF) polymerase chain reaction (PCR) result for either EV or HPeV RNA, using assays previously described elsewhere, ${ }^{2,4}$ which were performed as part of the routine workup for neonates or infants admitted with suspected sepsis. Additional samples were taken depending on the degree of clinical illness, including: EDTA blood, rectal swabs or stool samples, and various respiratory samples (nasopharyngeal aspirates, throat swabs and bronchoalveolar lavages). For each patient, their laboratory parameters were extracted from the laboratory database and clinical notes were reviewed.

This study was performed as part of a paediatric departmental audit which aimed to ensure that all $\mathrm{EV}$ and $\mathrm{HPeV}$-infected paediatric patients had received appropriate follow-up after discharge for sepsis, where there was laboratory confirmed EV or HPeV infection of the CSF. Therefore formal ethics approval was not required.

Clinical parameters examined included: age at presentation, length of stay, fever, rash, seizures, respiratory difficulty, feeding problems, antimicrobial use, and admission to high dependency or intensive care units. Laboratory parameters compared included: C-reactive protein (CRP), white cell counts (WCC), liver function tests (LFT), CSF profile (glucose, protein, and cell counts), and radiological investigations, where available.

Clinical and laboratory characteristics were compared between patients with $\mathrm{HPeV}$ and EV infection. Continuous variables were presented as mean and standard deviation (or median and interquartile range if not normally distributed) and compared with Student t-test (or Wilcoxon test). Binary variables were presented as frequency and percentages, and compared with the Fisher Exact test. Multivariable analysis for risk ratios (RR) comparing $\mathrm{HPeV}$ infection to $\mathrm{EV}$ infection as the reference, were estimated using log-Binomial regression model. 


\section{RESULTS}

There were no statistically significant differences in age or sex of the children affected by EV vs HPeV CNS infections but there appeared to be a difference in range, with EV often affecting older children than HPeV (IQR 29-102 days for EV and 25.5-61 days for $\mathrm{PeV})$.

Out of a total of 163 cases, there were 131 EV (i.e. 7 Coxsackie A, 18 Coxsackie B, 46 echoviruses, with 60 enteroviruses that could not be typed further) and $32 \mathrm{HPeV}$ infections (Table 1). All HPeV infections were caused by HPeV genotype 3 (HPeV-3). Of the EV cases, $73 \%$ (95 cases) were in children younger than 90 days (3 months), whereas over $90 \%$ (30 cases) of $\mathrm{HPeV}$ cases were in children younger than 90 days (3 months).

Cases of enterovirus meningitis showed three peaks of activity each year with the most significant being in the Nov-Dec period. In contrast, $\mathrm{HPeV}$ had only one significant outbreak over two months in summer 2016 (Figure S1). There was no difference in the mean age or sex of the children affected by EV or PeV, although there was a difference in range, with EV meningitis affecting some much older children.

A greater number of abnormal parameters were found with $\mathrm{HPeV}$ than for enteroviruses, with a greater likelihood of admission to high dependency unit (HDU)/ intensive care unit (ICU) $(\mathrm{p}=0.004)$ and a higher rate of persistent symptoms (i.e. fever, irritability, and feeding problems, $\mathrm{p}<0.05$ ) (Table 2). Compared with children infected with $\mathrm{EV}$, children with $\mathrm{HPeV}$ were more likely to have an abnormally low WCC (leukopenia) (56\% $\mathrm{HPeV}$ vs. $14 \% \mathrm{EV}, \mathrm{p}<0.001$ ), and an abnormally low lymphocyte count (lymphopenia) (91\% HPeV vs. 39\% EV, p<0.001) (Table S1).

In contrast, EV cases were more likely to have a high white cell count in the CSF $(6 \%$ $\mathrm{HPeV}$ vs. 50\% EV, p<0.001) (Table S1). In the adjusted (log-Binomial regression) analysis, the $\mathrm{HPeV}$ cases were over 5 more times more likely to have lymphopenia than EV cases 
$(\mathrm{RR}=5.11,95 \% \mathrm{CI} 1.53-17.05, \mathrm{p}=0.008)$, with $\mathrm{EV}$ cases being marginally more likely to have a higher CSF WCC $(\mathrm{RR}=0.22,95 \%$ CI $0.05-0.92, \mathrm{p}=0.038)$ (Table S2).

Other laboratory and clinical parameters, including overall length of stay (LOS) did not differ significantly between the EV or $\mathrm{HPeV}$ cases, however. There was no significant difference in whether or not $\mathrm{EV}$ or $\mathrm{HPeV}$ cases received antibiotic $(98.4 \%$ vs. $100 \%$, respectively, $\mathrm{p}=0.999)$ and/or acyclovir $(37.1 \%$ vs. $34.4 \%$, respectively, $\mathrm{p}=0.839)$ treatment (Table 2).

Finally, relatively few patients were deemed to require longer-term followup. Of the total number of cases, $80 \%$ of children did not require any follow-up at one year postinfection. At one year post-infection 3\% of children were under follow-up by ophthalmology, with no abnormalities detected. Sixteen children $(\sim 10 \%)$ attended a routine hearing check but none had any detectable sensorineural hearing loss. Only 3 patients $(<2 \%)$ were reported as having had any developmental delay problems on admission: 1 child had delayed speech and manipulative skills - both of which resolved by one year post-infection. Another child still had some speech delay at one year followup, and one child had gross motor delay (despite a normal MRI). As 2 of the 3 children with developmental problems had uncomplicated, short inpatient stays, this might suggest that their viral infection were not direct causes of this. However, this does not completely exclude this aetiological possibility.

\section{DISCUSSION}

Infections by EVs and HPeVs are well-documented causes of neonatal and infant sepsis. However relatively few studies have compared the severity of clinical illness caused by these viruses within the same paediatric population within the same season. ${ }^{5}$

Here we demonstrate differences in presentation and severity of these two viruses, with $\mathrm{HPeV}$ cases having a higher likelihood of having persistent fevers $(\mathrm{p}<0.05)$, irritability 
or feeding problems $(\mathrm{p}<0.05)$, leukopenia, lymphopenia, and requiring admission to HDU or PICU than children with EV infections. These findings are consistent with those reported from other studies. ${ }^{6-8}$ In addition, more specifically, Cabrerizo and colleagues ${ }^{9}$ also noted a higher CSF pleocytosis in EV vs. HPeV infections, as found in this study.

In our population more children aged 30-90 days $(n=19,58 \%)$ were infected with $\mathrm{HPeV}$ than neonates $(n=11,33 \%)$. Some studies have found children over the age of 2 months $^{8}$ or 3 months ${ }^{9}$ were unaffected by $\mathrm{HPeV}$, whereas $21 \%$ of our cases ( 7 patients) were diagnosed in children aged over 2 months, with 2 cases being in a 4-month and 6-month-old, respectively. Thus, routine testing for $\mathrm{HPeV}$ in all children with febrile rash illness and sepsis may reveal a higher number of older children infected with $\mathrm{HPeV}$.

Although some previous studies have found pediatric $\mathrm{HPeV}$ and $\mathrm{EV}$ infections clinically indistinguishable, ${ }^{1,3}$ anecdotally, in our pediatric population, nurses who worked with children involved in our recent $\mathrm{HPeV}$ outbreak, ${ }^{2}$ reported that they were able to distinguish which children had $\mathrm{HPeV}$ rather than EV, prior to any laboratory confirmation, on their clinical presentation alone. These $\mathrm{HPeV}$ cases were noted to be generally more irritable and persistently unconsolable, tachychardic and pyrexial than the more frequently encountered annual, seasonal EV cases with which the nurses were very familiar.

The main limitation of this study is related to the infrequent and sporadic approach to the longer-term followup of these $\mathrm{EV}$ and $\mathrm{HPeV}$-infected patients, as individual clinical teams were left to decide on whether patients being admitted under them warranted such followup. This was mostly based on the individual patient's clinical course during their admission, as well as prior experiences of the lead paediatrician concerned, rather than any local consensus guidelines.

At present clinical guidelines do not differentiate between the management of children presenting with $\mathrm{EV}$ versus $\mathrm{HPeV}$ infections. ${ }^{3}$ This study demonstrates that 
differences in the severity of clinical illness can be seen between the HPeV and EV CNS infections, with a greater degree of severity in $\mathrm{HPeV}$ cases. Further studies are required to clarify and confirm these findings, which may then lead to more practical clinical guidelines for the immediate and longer-term management and followup of these patients.

\section{Word count 1493}

\section{REFERENCES}

1. Verboon-Maciolek MA, Krediet TG, Gerards LJ, et al. Severe neonatal parechovirus infection and similarity with enterovirus infection. Pediatr Infect Dis J. 2008;27:241245.

2. Tang JW, Holmes CW, Elsanousi FA, et al. Cluster of human parechovirus infections as the predominant cause of sepsis in neonates and infants, Leicester, United Kingdom, 8 May to 2 August 2016. Euro Surveill. 2016;21:pii=30326.

3. Drysdale SB, Kelly DF. Fifteen-minute consultation: enterovirus meningitis and encephalitis—when can we stop the antibiotics? Arch Dis Child Educ Pract Ed. 2017;102:66-71.

4. Holmes CW, Koo SS, Osman H, et al. Predominance of enterovirus B and echovirus 30 as cause of viral meningitis in a UK population. J Clin Virol. 2016;81:90-93.

5. Walters B, Peñaranda SW, Nix AM et al. Detection of human parechovirus (HPeV)-3 in spinal fluid specimens from pediatric patients in the Chicago area. J Clin Virol. 2011;52:187-191. 
6. Sharp J, Harrison CJ, Puckett K, et al. Characteristics of young infants in whom human parechovirus, enterovirus or neither were detected in cerebrospinal fluid during sepsis evaluations. Pediatr Infect Dis J. 2013;32:213-216.

7. Sano K, Hamada H, Hirose S, et al. Prevalence and characteristics of human parechovirus and enterovirus infections in febrile infants. Pediatr Int. 2017 Dec 5. Doi:10.1111/ped.13467. [Epub ahead of print]

8. Harvala H, Calvert J, Van Nguyen D, et al. Comparison of diagnostic clinical samples and environmental sampling for enterovirus and parechovirus surveillance in Scotland, 2010 to 2012. Euro Surveill. 2014;19:pii=20772.

9. Cabrerizo M, Trallero G, Pena MJ, Cilla A, et al. Comparison of epidemiology and clinical characteristics of infections by human parechovirus vs. those by enterovirus during the first month of life. Eur J Pediatr. 2015;174:1511-1516. 
Table 1. Specific human enteroviruses (EVs) by type and proportion, identified in the CSF of these patients

\begin{tabular}{|c|c|c|}
\hline EVs & Number & Percentage (\%) \\
\hline CA16 & 1 & 0.76 \\
\hline CA6 & 2 & 1.53 \\
\hline CA9 & 4 & 3.05 \\
\hline CB1 & 1 & 0.76 \\
\hline CB4 & 7 & 5.34 \\
\hline CB5 & 10 & 7.63 \\
\hline E11 & 2 & 1.53 \\
\hline E16 & 3 & 2.29 \\
\hline E18 & 7 & 5.34 \\
\hline E21 & 1 & 0.76 \\
\hline E25 & 3 & 2.29 \\
\hline E3 & 1 & 0.76 \\
\hline E30 & 3 & 2.29 \\
\hline E5 & 5 & 3.82 \\
\hline E6 & 4 & 3.05 \\
\hline E7 & 5 & 3.82 \\
\hline E71 & 1 & 0.76 \\
\hline E9 & 10 & 7.63 \\
\hline EV7 & 1 & 0.76 \\
\hline Untypeable & 60 & 45.80 \\
\hline Total & 131 & 100.00 \\
\hline
\end{tabular}


TABLE 2. Clinical and laboratory characteristics of the EV and HPeV cases

\section{Enterovirus $\quad$ Parechovirus p-value \\ (EV) $(\mathrm{n}=131) \quad(\mathrm{HPeV})(\mathrm{n}=32)$}

\section{Demographics}

\begin{tabular}{|c|c|c|c|}
\hline Age (days) & $50(29-102)$ & $39.5(25.5-61)$ & 0.069 \\
\hline Sex (female) & $42.0 \%(55 / 131)$ & $40.6 \%(13 / 32)$ & 0.999 \\
\hline \multicolumn{4}{|l|}{ Symptoms } \\
\hline Fever & $94.6 \%(123 / 130)$ & $100 \%(32 / 32)$ & 0.347 \\
\hline Peak temperature $\left({ }^{\circ} \mathrm{C}\right)$ & $38.5(0.7)$ & $38.7(0.7)$ & 0.293 \\
\hline Feeding problems & $56.6 \%(73 / 129)$ & $68.8 \%(22 / 32)$ & 0.234 \\
\hline Rash in history & $25.2 \%(32 / 127)$ & $25.0 \%(8 / 32)$ & 0.999 \\
\hline Seizure & $4.7 \%(6 / 127)$ & $3.1 \%(1 / 32)$ & 0.999 \\
\hline \multicolumn{4}{|l|}{ Respiratory symptoms (coryzal } \\
\hline symptoms, grunting, cough, wheeze). & $35.2 \%(45 / 128)$ & $37.5 \%(12 / 32)$ & 0.838 \\
\hline \multicolumn{4}{|l|}{ Blood and CSF results } \\
\hline \multicolumn{4}{|l|}{ C-reactive protein $(\mathrm{CRP}, \mathrm{mg} / \mathrm{L})$} \\
\hline (normal range: 0 - 10) & $10(3-24)$ & $6(3-13.5)$ & 0.073 \\
\hline \multicolumn{4}{|l|}{ White cell count $\left(\mathrm{WCC}, \mathrm{x} 10^{9} / \mathrm{L}\right)$} \\
\hline (normal range: $6.0-17.0$ ) & $10.5(4.1)$ & $6.4(2.9)$ & $<\mathbf{0 . 0 0 1}$ \\
\hline \multicolumn{4}{|l|}{ Neutrophils (x109/L) } \\
\hline (normal range: $1.50-8.50$ ) & $5.5(3.4)$ & $3.7(2.5)$ & $<0.001$ \\
\hline \multicolumn{4}{|l|}{ Lymphocytes $\left(\mathrm{x} 10^{9} / \mathrm{L}\right)$} \\
\hline (normal range: $4.00-13.50$ ) & $4.0(2.1)$ & $2.0(0.8)$ & $<\mathbf{0 . 0 0 1}$ \\
\hline \multicolumn{4}{|l|}{ Platelets $\left(\mathrm{x} 10^{9} / \mathrm{L}\right)$} \\
\hline (normal range: 140 - 400) & $390.1(130.0)$ & $353.7(139.1)$ & 0.165 \\
\hline Alanine transferase (ALT, IU/L) & $23(18-30)$ & $24(20-31)$ & 0.465 \\
\hline
\end{tabular}


(normal range: 2 - 53)

Total bilirubin $(\mu \mathrm{mol} / \mathrm{L})$

(normal range: 0 - 21)

$10.5(5-22)$

$12(8-32)$

0.284

CSF glucose (mmol/L)
$2.9(2.6-3.2)$
$3.1(2.75-3.2)$
0.123

CSF protein $(\mathrm{g} / \mathrm{L})$

(normal range: $0.10-0.45$ )

$0.45(0.32-0.65) \quad 0.39(0.31-0.67) \quad 0.338$

CSF RBC (x10 $/ \mathrm{L})$

(normal range: 0)

$8(2-500)$

$4(1-685)$

0.481

CSF WCC (x106/L)

(normal range: 0-20)

$5(1-75)$

$1(0-2)$

$<\mathbf{0 . 0 0 1}$

CSF \%polymorphs ${ }^{*}$

(not applicable)

$12(5-40)$

CSF \%lymphocytes

(not applicable)

$76.5(54-88)$

\begin{tabular}{llll}
\hline CSF taken before antibiotics given & $61.9 \%(78 / 126)$ & $43.8 \%(14 / 32)$ & 0.073
\end{tabular}

\section{Treatment and outcome}

\begin{tabular}{lllc}
\hline Antibiotics given during admission & $98.4 \%(127 / 129)$ & $100 \%(32 / 32)$ & 0.999 \\
\hline Acyclovir given during admission & $37.1 \%(46 / 124)$ & $34.4 \%(11 / 32)$ & 0.839 \\
\hline Persistent ${ }^{* *}$ pyrexia (first 24-48 hours) & $33.1 \%(42 / 127)$ & $53.1 \%(17 / 32)$ & $\mathbf{0 . 0 4 2}$ \\
\hline Persistent ${ }^{* *}$ irritability/feeding problem & $19.0 \%(24 / 126)$ & $37.5 \%(12 / 32)$ & $\mathbf{0 . 0 3 4}$ \\
\hline At least 1 seizure post antibiotic & & & \\
treatment & $2.4 \%(3 / 127)$ & $6.3 \%(2 / 32)$ & 0.264 \\
\hline Length of stay (LOS, days) & $4(3-5)$ & $4(3-5)$ & 0.680 \\
\hline PICU/HDU & $6.1 \%(8 / 131)$ & $25.0 \%(8 / 32)$ & $\mathbf{0 . 0 0 4}$ \\
\hline LOS PICU/HDU*** & $2(1-5)$ & $2.5(1-4.5)$ & 0.906
\end{tabular}

Footnotes: PICU - pediatric intensive care; HDU high dependency unit. 
Continuous data expressed as mean (SD) or median (Q1-Q3) as appropriate, and binary as \% (n).

Missing data - peak temperature (4), WCC (4), platelets (4), neutrophils (5), lymphocytes (5), ALT (34), bilirubin (34), CSF protein (2), CSF RBC (3), CSF WCC (3)

*data available for: 53 patients for CSF \%polymorphs; 54 patients for CSF \%lymphoctyes

**, Persistent' indicating that it has continued for 24-48 hours after treatment commenced

${ }^{* * *}$ PICU/HDU LOS available in 15 patients (7 EV and $\left.8 \mathrm{HPeV}\right)$ 